\title{
Comparison of Vibration and Acoustic Pressure Reduction Using Different Types of Piezo actuators
}

\author{
J. WiCiak* AND R. TROJANOWSKi \\ AGH University of Science and Technology, Department of Mechanics and Vibroacoustics, \\ Al. A. Mickiewicza 30, 30-059 Krakow, Poland
}

\begin{abstract}
This paper presents results of numerical simulations on reduction of sound pressure radiated from a square plate using different types of piezo actuators. For this purpose a FEM model was developed using ANSYS software with said steel plate with a hemisphere of air on one side of the plate. There are two criteria by which the reduction is measured: minimising vibrations of plate area and minimising sound pressure on a half sphere surrounding the plate. Changes in the results for both the radiated sound pressure and plate vibration are discussed.
\end{abstract}

DOI: 10.12693/APhysPolA.128.A-62

PACS: 43.40.-r, 43.28.Js, 46.40.Ff

\section{Introduction}

Beam, shell or plate structures are typical objects used in theoretical and experimental analyses of structureborne sound and vibrations. First of all, they have fairly simple geometry which makes it easier to describe their behaviour under external factors analytically $[1,2]$. Using mathematical models of beams an analytical solutions in active vibration protection and optimal placement of actuators can be found $[3,4]$. Also, estimations of sound radiated from plate structures can be calculated $[2,5,6]$. On the other hand, more complicated systems are usually composed of these simple elements and analyses and experiments made on them can be later implemented to complex structures. Therefore they can be also used to verify analytical $[7,8]$, or numerical $[9,10]$ solutions.

This paper presents results of numerical analyses concerning differences in reduction of sound radiated from a steel plate when using different shapes and composition of piezo actuators.

Previous publications that led to this paper first started with analytical and numerical [11] modelling of a circular plate with homogeneous square- and disc-shaped piezo actuators attached. After that a numerical study on vibration reduction of square plate using different shapes (square and disc) and composition of piezo elements [12] has been carried out. It was the present authors' first journal publication in which a concept of a two-part element was tested. The next step was testing if the placement of the inner part of a two-part element had an effect on its effectiveness [13]. Another publication [14] expanded on numerical models and presented early results of physical experiment.

Results presented in this paper are based on models created using ANSYS software presented in $[12,14]$ with addition of a hemisphere of air. Modelled air is used for

* corresponding author; e-mail: wiciak.agh.edu.pl calculating sound pressure reduction using two criteria - minimising vibrations of the area of the plate or minimising sound pressure on a half sphere with a radius of $0.5 \mathrm{~m}$.

\section{Numerical models}

The plate model is actually the same as in $[12,14]$ that is a steel plate $(400 \mathrm{~mm} \times 400 \mathrm{~mm} \times 2 \mathrm{~mm})$ with 2 piezo elements attached. One of these elements is a homogeneous square-based element placed near the centre of the plate with area of $1600 \mathrm{~mm}^{2}$ and height of $1 \mathrm{~mm}$ used to excite vibration of the plate. The other one differs in shape and material composition (with the same area of $1600 \mathrm{~mm}^{2}$ and height of $1 \mathrm{~mm}$ ) and is used to reduce plate vibrations. Figure 1a shows the arrangement of elements on the plate. As this paper will deal with acoustic pressure radiated from the plate and its reduction it was necessary to expand the model by adding surrounding air. This was done by modelling a hemisphere of air with a radius of $1 \mathrm{~m}$. The boundaries of said half ball were modelled as to have full absorption. Therefore the modelled volume can be treated as a part of the open half space. The full model is presented in Fig. 1b.
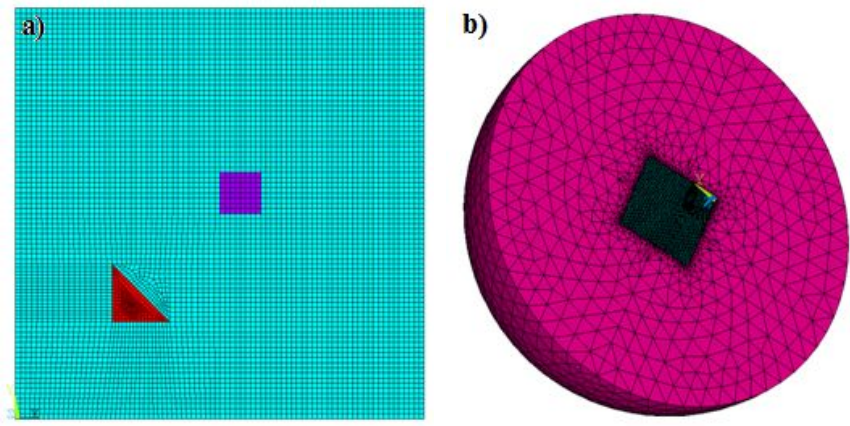

Fig. 1. The arrangement of piezo elements on the plate (a) and full model (b). 
Because different shapes of actuators resulted in different mesh essentially three models were created. The modelled actuator shapes included a square-based, a disc-based and a right-angled triangle-based element. As it was mentioned before they always had the same base surface area of $1600 \mathrm{~mm}^{2}$ and height of $1 \mathrm{~mm}$. The material composition of these elements could be either homogeneous modelled using properties of PZ28 or PZ29, or a two-part composition with the inner part made of one material and the outer part made of the other. The base area of the inner part made $1 / 4$ of the base area of the whole element. This gives four cases for each shape: a homogeneous PZ28, a homogeneous PZ29, a two-part element with the inner part made of PZ28 and the outer part of PZ29 (referred to in the following as PZ28/PZ29), and a two-part element with the inner part made of PZ29 and the outer part of PZ28 (PZ29/PZ28). The modelled two part elements are shown in Fig. 2.
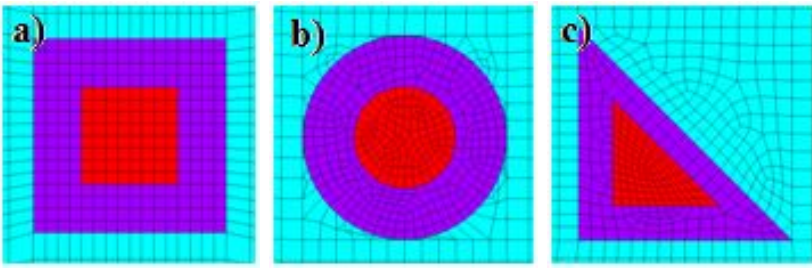

Fig. 2. The modelled piezo elements: square-based; (b) two-part disc-based; (a) two-part right-angled triangle-based.

Table I shows which elements were used when developing the models together with material properties that were assigned to them.

TABLE I

Model parameters.

\begin{tabular}{c|c|c}
\hline \hline Structural elements & $\begin{array}{c}\text { Element used } \\
\text { for modelling }\end{array}$ & Material properties \\
\hline Plate & SOLSH190 & $\begin{array}{c}E=1.93 \times 10^{11} \mathrm{~Pa}, \\
\nu=0.29, \\
\rho=7800 \mathrm{~kg} / \mathrm{m}^{3}\end{array}$ \\
\hline $\begin{array}{c}\text { Piezo element used } \\
\text { for plates excitation }\end{array}$ & SOLID226 & Properties of PZ28 \\
\hline Actuators & SOLID226 & $\begin{array}{c}\text { Combination of proper- } \\
\text { ties of PZ28 and PZ29 }\end{array}$ \\
\hline Air & FLUID30 & $\begin{array}{c}\rho_{c}=1.2 \mathrm{~kg} / \mathrm{m}^{3}, \\
c=343 \mathrm{~m} / \mathrm{s}\end{array}$
\end{tabular}

Modal analyses were performed to find the first six modes after which modes 1 and 5 were selected for further analyses. Mode 1 was chosen as a fundamental mode, and mode 5 was chosen because the placement of the second actuator is close to it's antinode. For harmonic analyses, a voltage of $100 \mathrm{~V}$ was applied to the element responsible for plate excitation. The search for optimal amplitudes and phases of voltage for actuators was done using internal ANSYS optimisation procedures. There were 2 goal functions. The first goal function was assumed in the form

$$
J_{1}=\min \sum_{i=1}^{n}\left|\mathbf{X}_{\text {sum }}(i)\right|,
$$

where $\mathbf{X}_{\text {sum }}(i)$ is displacement vector in $i$-th node and $n$ is number of nodes (in this case all nodes making up the base of the plate: 7654 nodes with squarebased elements, 7296 nodes with disc-based elements and 6932 with triangle-based elements).

The form of the second goal function was:

$$
J_{2}=\min \sum_{j=1}^{m}|p(j)|,
$$

where $|p(j)|$ is the absolute value of sound pressure in $j$-th node and $m$ is the number of nodes (in this case, all nodes making up the hemisphere with $r=0.5: 186$ nodes with square-based elements, 186 nodes with disc-based elements and 194 nodes with triangle-based elements).

The sound pressure radiated from the plate can be expressed by means Huygens-Rayleigh formula [15, 16]:

$$
p(\boldsymbol{r})=\frac{-i k}{2 \pi} \rho_{c} c \int_{S_{0}} v\left(\boldsymbol{r}_{0}\right) \frac{\exp \left(i \boldsymbol{k}\left(\boldsymbol{r}-\boldsymbol{r}_{0}\right)\right)}{\left|\boldsymbol{r}-\boldsymbol{r}_{0}\right|},
$$

where, $k$ is the wave number, $\rho_{c}$ is the density of medium, $c$ is the sound speed in medium, $S_{0}$ is the area of sound source, $v\left(\boldsymbol{r}_{0}\right)$ is the normal component of amplitude of velocity of vibrations of a point on source area, $\boldsymbol{k}$ is the wave vector, and $\left|\boldsymbol{r}-\boldsymbol{r}_{0}\right|=\sqrt{\left(x-x_{0}\right)^{2}+\left(y-y_{0}\right)^{2}+z^{2}}$.

It has been readily determined that the optimal phase of voltage applied to the piezo element used for reduction of the first mode was $180^{\circ}$ and $0^{\circ}$ for reduction of the fifth mode. That left only the amplitude of the voltage applied to actuator as a design variable. The number of steps performed during one optimisation procedure was chosen to be 30. After each completion of the procedure, it was repeated with the amplitude range being narrowed. In the final run, the range was $5 \mathrm{~V}$.

\section{Results}

This section presents results for the vibration and sound pressure reduction. Tables II-IV show results obtained when using the $J_{1}$ type goal function, and Tables V-VII present results obtained using goal function $J_{2}$.

The vibration reduction $\mathrm{L}_{\text {red }}$ was calculated as:

$$
\mathrm{L}_{\mathrm{red}}=20 \log \frac{\sum_{i=1}^{n}\left|\mathbf{X}_{1 \text { sum }}(i)\right|}{\sum_{i=1}^{n}\left|\mathbf{X}_{2 \text { sum }}(i)\right|},
$$

where $\mathbf{X}_{1 \text { sum }}(i)$ is the displacement vector in $i$-th node before reduction, $\mathbf{X}_{2 \text { sum }}(i)$ is the displacement vector in $i$-th node after reduction and $n$ is the number of nodes making up the area of the plate (the same as in Eq. 1).

The reduction of the radiated sound pressure $L_{\text {pred }}$ was calculated as: 


$$
\mathrm{L}_{\text {pred }}=20 \log \frac{\sum_{j=1}^{m}\left|p_{1}(j)\right|}{\sum_{j=1}^{m}\left|p_{2}(j)\right|},
$$

where $p_{1}(j)$ is the pressure in $j$-th node before reduction, $p_{2}(j)$ is the pressure in $j$-th node after reduction and $m$ is the number of nodes on the hemisphere with $r=0.5 \mathrm{~m}$.

Numerical simulations carried out using the goal function $J_{1}$ presented in Tables II-IV show no significant differences between elements with different material composition - (less than $1 \mathrm{~dB}$ ). These differences are independent of both actuator shape and mode shape.

There were differences between different shapes of actuators. If square-based actuators are used as a reference, it can be seen that for the first mode the vibration reduction obtained using disc-based elements is up to $1.5 \mathrm{~dB}$ lower, and using triangle-based actuators resulted in higher reduction of up to $2.3 \mathrm{~dB}$. The results for the fifth mode show smaller differences (less than $1 \mathrm{~dB}$ for disc-based actuator and less than $1.1 \mathrm{~dB}$ for trianglebased actuator).

\section{TABLE II}

Results for the square-based piezo actuators using the goal function $J_{1}$ : material - the material used for inner/outer part of actuator; mode — the mode shape; $\mathrm{U}$ - amplitude of voltage applied to the actuator; $\phi-$ phase angle of voltage applied to the actuator; $L_{\text {red }}$ vibration reduction level obtained from the simulation; $\mathrm{L}_{\mathrm{pred}}$ - the sound pressure reduction level obtained from the simulation.

\begin{tabular}{c|c|c|c|c|c}
\hline \hline $\begin{array}{c}\text { Material } \\
\text { inner/outer }\end{array}$ & Mode & $\begin{array}{c}\mathrm{U} \\
{[\mathrm{V}]}\end{array}$ & $\begin{array}{c}\phi \\
{\left[{ }^{\circ}\right]}\end{array}$ & $\begin{array}{c}\mathrm{L}_{\text {red }} \\
{[\mathrm{dB}]}\end{array}$ & $\begin{array}{c}\mathrm{L}_{\text {pred }} \\
{[\mathrm{dB}]}\end{array}$ \\
\hline PZ 28 & 1 & 365.29 & 180.00 & 41.3 & 56.2 \\
PZ 29/PZ 28 & 1 & 294.88 & 180.00 & 41.3 & 55.0 \\
PZ 29 & 1 & 198.29 & 180.00 & 41.0 & 52.8 \\
PZ 28/PZ 29 & 1 & 228.29 & 180.00 & 41.3 & 56.2 \\
PZ 28 & 5 & 159.95 & 360.00 & 34.7 & 50.4 \\
PZ 29/PZ 28 & 5 & 129.10 & 360.00 & 35.0 & 46.6 \\
PZ 29 & 5 & 86.93 & 360.00 & 34.6 & 49.8 \\
PZ 28/PZ 29 & 5 & 100.45 & 360.00 & 34.4 & 49.5 \\
& & & & &
\end{tabular}

Results for vibration reduction obtained when using the goal function $J_{2}$ (Tables V-VII) are even more similar - this time the differences between elements with different material composition are lower than $0.3 \mathrm{~dB}$.

Differences resulting from different shapes of actuators are actually quite similar to those obtained when using the goal function $J_{1}$. Again for the first mode the difference is up to $1.5 \mathrm{~dB}$ between square- and disc-based actuators, and up to $2.3 \mathrm{~dB}$ between triangle-based and square-based actuators. For the fifth mode the difference is up to $0.6 \mathrm{~dB}$ between disc-based and square-based actuators, and up to $1 \mathrm{~dB}$ between triangle-based and square-based actuators.

Interpreting the results for the sound pressure reduction (Tables II-VII) it can be seen that when minimisation of plate vibration is used as a criterion (function $J_{1}$ ),

\section{TABLE III}

Results for the disc-based piezo actuators using the goal function $J_{1}$ : material - the material used for inner/outer part of actuator; mode - the mode shape; U - amplitude of voltage applied to the actuator; $\phi-$ phase angle of voltage applied to the actuator; $\mathrm{L}_{\mathrm{red}}$ - vibration reduction level obtained from the simulation; $\mathrm{L}_{\text {pred }}$ the sound pressure reduction level obtained from the simulation.

\begin{tabular}{c|c|c|c|c|c}
\hline \hline $\begin{array}{c}\text { Material } \\
\text { inner/outer }\end{array}$ & Mode & $\begin{array}{c}\mathrm{U} \\
{[\mathrm{V}]}\end{array}$ & $\begin{array}{c}\phi \\
{\left[{ }^{\circ}\right]}\end{array}$ & $\begin{array}{c}\mathrm{L}_{\text {red }} \\
{[\mathrm{dB}]}\end{array}$ & $\begin{array}{c}\mathrm{L}_{\text {pred }} \\
{[\mathrm{dB}]}\end{array}$ \\
\hline PZ 28 & 1 & 371.94 & 180.00 & 39.9 & 56.4 \\
PZ 29/PZ 28 & 1 & 300.24 & 180.00 & 39.8 & 55.1 \\
PZ 29 & 1 & 200.24 & 180.00 & 39.8 & 54.4 \\
PZ 28/PZ 29 & 1 & 230.81 & 180.00 & 39.9 & 54.9 \\
PZ 28 & 5 & 161.98 & 360.00 & 35.1 & 47.0 \\
PZ 29/PZ 28 & 5 & 131.24 & 360.00 & 34.8 & 47.4 \\
PZ 29 & 5 & 87.29 & 360.00 & 35.2 & 47.9 \\
PZ 28/PZ 29 & 5 & 101.24 & 360.00 & 35.3 & 49.3 \\
& & & & &
\end{tabular}

TABLE IV

Results for the right-angled triangle-based piezo actuators using the goal function $J_{1}$ : material - the material used for inner/outer part of actuator; mode - the mode shape; U - amplitude of voltage applied to the actuator; $\phi-$ phase angle of voltage applied to the actuator; $\mathrm{L}_{\mathrm{red}}$ - vibration reduction level obtained from the simulation; $\mathrm{L}_{\mathrm{pred}}$ - the sound pressure reduction level obtained from the simulation.

\begin{tabular}{c|c|c|c|c|c}
\hline $\begin{array}{c}\text { Material } \\
\text { inner/outer }\end{array}$ & Mode & $\begin{array}{c}\mathrm{U} \\
{[\mathrm{V}]}\end{array}$ & $\begin{array}{c}\phi \\
\left.{ }^{\circ}\right]\end{array}$ & $\begin{array}{c}\mathrm{L}_{\text {red }} \\
{[\mathrm{dB}]}\end{array}$ & $\begin{array}{c}\mathrm{L}_{\text {pred }} \\
{[\mathrm{dB}]}\end{array}$ \\
\hline PZ 28 & 1 & 255.02 & 180.00 & 43.6 & 58.4 \\
PZ 29/PZ 28 & 1 & 212.02 & 180.00 & 42.9 & 53.8 \\
PZ 29 & 1 & 145.55 & 180.00 & 43.3 & 58.1 \\
PZ 28/PZ 29 & 1 & 165.55 & 180.00 & 43.2 & 58.4 \\
PZ 28 & 5 & 201.24 & 360.00 & 35.5 & 29.2 \\
PZ 29/PZ 28 & 5 & 156.94 & 360.00 & 35.0 & 31.7 \\
PZ 29 & 5 & 109.24 & 360.00 & 35.0 & 27.3 \\
PZ 28/PZ 29 & 5 & 127.69 & 360.00 & 35.5 & 28.0 \\
& & & & &
\end{tabular}

differences in the obtained reduction can be rather significant. For square-based elements the difference is up to $3.4 \mathrm{~dB}$ for the first mode and 3.8 for the fifth mode. For disc-based actuators, the difference was up to $2 \mathrm{~dB}$ for the first mode and $2.3 \mathrm{~dB}$ for the fifth mode. And finally, for triangle-based actuators the difference was up to $4.6 \mathrm{~dB}$ for the first mode and $4.4 \mathrm{~dB}$ for the fifth mode. However when changing the optimisation criterion to minimising the sound pressure around the plate (function $J_{2}$ ), these differences grow smaller not exceeding $0.6 \mathrm{~dB}$. Also in all cases sound pressure reduction obtained using the goal function $\mathrm{J}_{2}$ are higher than those obtained with the goal function $J_{1}$.

Different shapes of actuators show some interesting features as far as the sound pressure reduction is considered. Although the triangle-shaped actuators show greater reduction than square- or disc- based actuators 


\section{TABLE V}

Results for the square-based piezo actuators using the goal function $J_{2}$ : material - the material used for inner/outer part of actuator; mode - the mode shape; $\mathrm{U}$ - amplitude of voltage applied to the actuator; $\phi-$ phase angle of voltage applied to the actuator; $\mathrm{L}_{\mathrm{red}}$ vibration reduction level obtained from the simulation; $\mathrm{L}_{\text {pred }}$ - the sound pressure reduction level obtained from the simulation.

\begin{tabular}{c|c|c|c|c|c}
\hline \hline $\begin{array}{c}\text { Material } \\
\text { inner/outer }\end{array}$ & Mode & $\begin{array}{c}\mathrm{U} \\
{[\mathrm{V}]}\end{array}$ & $\begin{array}{c}\phi \\
{\left[{ }^{\circ}\right]}\end{array}$ & $\begin{array}{c}\mathrm{L}_{\text {red }} \\
{[\mathrm{dB}]}\end{array}$ & $\begin{array}{c}\mathrm{L}_{\text {pred }} \\
{[\mathrm{dB}]}\end{array}$ \\
\hline PZ 28 & 1 & 365.20 & 180.00 & 41.3 & 56.5 \\
PZ 29/PZ 28 & 1 & 294.57 & 180.00 & 41.2 & 56.1 \\
PZ 29 & 1 & 197.96 & 180.00 & 41.3 & 56.5 \\
PZ 28/PZ 29 & 1 & 228.24 & 180.00 & 41.3 & 56.4 \\
PZ 28 & 5 & 159.76 & 360.00 & 34.9 & 50.5 \\
PZ 29/PZ 28 & 5 & 128.58 & 360.00 & 34.9 & 50.1 \\
PZ 29 & 5 & 86.98 & 360.00 & 34.8 & 49.9 \\
PZ 28/PZ 29 & 5 & 100.59 & 360.00 & 34.7 & 49.9
\end{tabular}

TABLE VI

Results for the disc-based piezo actuators using the goal function $J_{2}$ : material - the material used for inner/outer part of actuator; mode - the mode shape; U - amplitude of voltage applied to the actuator; $\phi-$ phase angle of voltage applied to the actuator; $\mathrm{L}_{\text {red }}$ - vibration reduction level obtained from the simulation; $L_{p r e d}-$ the sound pressure reduction level obtained from the simulation.

\begin{tabular}{c|c|c|c|c|c}
\hline $\begin{array}{c}\text { Material } \\
\text { inner/outer }\end{array}$ & Mode & $\begin{array}{c}\mathrm{U} \\
{[\mathrm{V}]}\end{array}$ & $\begin{array}{c}\phi \\
{\left[{ }^{\circ}\right]}\end{array}$ & $\begin{array}{c}\mathrm{L}_{\text {red }} \\
{[\mathrm{dB}]}\end{array}$ & $\begin{array}{c}\mathrm{L}_{\text {pred }} \\
{[\mathrm{dB}]}\end{array}$ \\
\hline PZ 28 & 1 & 371.83 & 180.00 & 39.9 & 56.5 \\
PZ 29/PZ 28 & 1 & 300.14 & 180.00 & 39.8 & 55.8 \\
PZ 29 & 1 & 199.94 & 180.00 & 39.8 & 55.9 \\
PZ 28/PZ 29 & 1 & 230.59 & 180.00 & 39.9 & 56.4 \\
PZ 28 & 5 & 162.60 & 360.00 & 35.4 & 50.3 \\
PZ 29/PZ 28 & 5 & 130.88 & 360.00 & 35.1 & 50.1 \\
PZ 29 & 5 & 87.51 & 360.00 & 35.4 & 50.2 \\
PZ 28/PZ 29 & 5 & 100.99 & 360.00 & 35.2 & 50.2
\end{tabular}

for the first mode (especially when using the goal function $J_{2}$ ), they are inferior when it comes to the reduction of the sound pressure generated by the fifth mode of the plate (up to $22.5 \mathrm{~dB}$ of difference when using the goal function $J_{1}$ ). The situation improves significantly when changing the optimisation criterion to this based on function $J_{2}$, but still it is up to $1.5 \mathrm{~dB}$ worse than using disc actuators. Another interesting feature is that while the vibration reduction level for the first mode and disc-based elements is worse than for the square-based ones, the sound pressure reduction is on a similar level.

One of the most interesting findings is the difference between the generated sound pressure reduction when using triangle based elements for the fifth mode. There is a significant improvement in the obtained reduction (ranging from $17.2 \mathrm{~dB}$ to $21.7 \mathrm{~dB}$ ), while the difference in the results for the plate vibration are rather small

\section{TABLE VII}

Results for the right-angled triangle-based piezo actuators using the goal function $J_{2}$ : material - the material used for inner/outer part of actuator; mode - the mode shape; U - amplitude of voltage applied to the actuator; $\phi-$ phase angle of voltage applied to the actuator; $\mathrm{L}_{\mathrm{red}}$ - vibration reduction level obtained from the simulation; $\mathrm{L}_{\text {pred }}$ - the sound pressure reduction level obtained from the simulation.

\begin{tabular}{c|c|c|c|c|c}
\hline $\begin{array}{c}\text { Material } \\
\text { inner/outer }\end{array}$ & Mode & $\begin{array}{c}\mathrm{U} \\
{[\mathrm{V}]}\end{array}$ & $\begin{array}{c}\phi \\
\left.{ }^{\circ}\right]\end{array}$ & $\begin{array}{c}\mathrm{L}_{\text {red }} \\
{[\mathrm{dB}]}\end{array}$ & $\begin{array}{c}\mathrm{L}_{\text {pred }} \\
{[\mathrm{dB}]}\end{array}$ \\
\hline PZ 28 & 1 & 254.88 & 180.00 & 43.6 & 59.4 \\
PZ 29/PZ 28 & 1 & 211.66 & 180.00 & 43.4 & 59.2 \\
PZ 29 & 1 & 145.60 & 180.00 & 43.4 & 59.0 \\
PZ 28/PZ 29 & 1 & 165.72 & 180.00 & 43.5 & 59.3 \\
PZ 28 & 5 & 194.45 & 360.00 & 35.7 & 48.8 \\
PZ 29/PZ 28 & 5 & 152.94 & 360.00 & 35.6 & 49.0 \\
PZ 29 & 5 & 104.70 & 360.00 & 35.6 & 49.0 \\
PZ 28/PZ 29 & 5 & 122.66 & 360.00 & 35.7 & 48.7
\end{tabular}

(up to $0.6 \mathrm{~dB}$ ). Figure 3 shows the results for the vibrations of the plate and sound pressure generated by it with and without reduction when using a triangle-based actuator with composition PZ29/PZ28. It can be seen that although vibrations of the plate did not change significantly their distribution did.
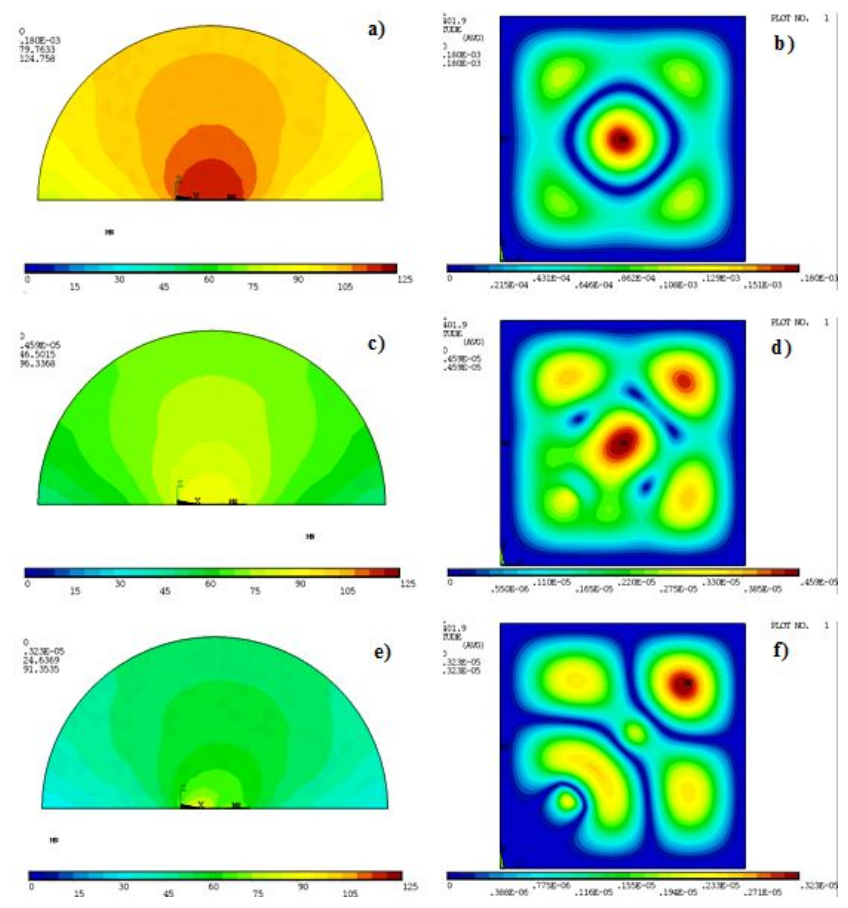

Fig. 3. Results for the fifth mode for triangle based piezo actuator with material composition of PZ29/PZ28: (a) sound pressure without reduction; (b) plate vibration without reduction; (c) sound pressure after reduction with function $J_{1}$; (d) plate vibration after reduction with function $J_{1}$; (e) sound pressure after reduction with function $J_{2}$, (f) plate vibration after reduction with $J_{2}$. 
Another interesting feature is shown in Fig. 4. When using the goal function of $J_{1}$ type the sound pressure generated by the plate is more or less symmetrical (Fig. 4a), but when using goal the function $J_{2}$ there is a part of space where the sound pressure level is about $15-20 \mathrm{~dB}$ lower (Fig. 4b). It could be therefore possible to obtain a local zone of silence by changing the goal function to focus on that part of the surrounding space.
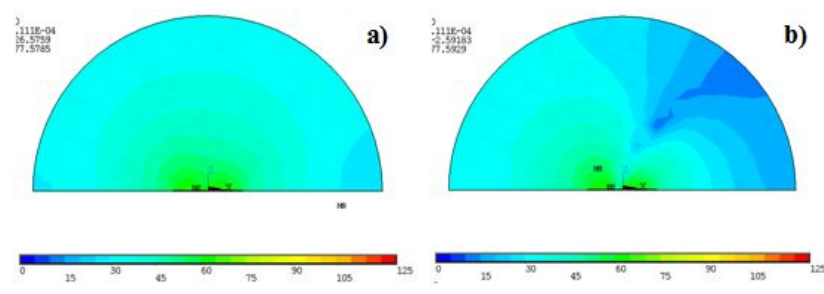

Fig. 4. Results for the first mode for the square based piezo actuator with material composition of PZ29:

(a) sound pressure after reduction with function $J_{1}$;

(b) sound pressure after reduction with function $J_{2}$.

When comparing the results obtained using goal functions $J_{1}$ and $J_{2}$ it can be seen that the reduction of the sound pressure level is improved every time, but for some cases such as e.g. for square-based actuator with material composition PZ29/PZ28 the vibration of the plate has increased.

If we take a look at the voltage applied to the actuators it can easily be seen that among the shapes chosen for the study, triangles were the most energy/voltage efficient.

\section{Conclusions}

The performed simulations show significant reduction in both vibrations of the plate and the radiated sound pressure.

Results of numerical simulation show that using piezo elements with a step change in material composition results in very small difference of both vibration and sound pressure level reduction when compared to homogeneous elements.

There were observed differences between the results of different shapes of actuators used.
Although not being the direct aim of this study, the use of the goal function $J_{2}$ (vibration reduction aimed at minimising the acoustical pressure radiated by the plate) has led to better results than using the goal function $J_{1}$ (vibration reduction aimed at minimising displacement of the back area of the plate). This was especially noticeable for the reduction of the fifth mode with triangle-based elements.

\section{Acknowledgments}

The project described in this paper has been executed within the project No. 11.11.130.955.

\section{References}

[1] M.S. Kozień, Acta Phys. Pol. A 123, 1029 (2013).

[2] M.S. Kozien, J. Theor. App. Mech. 47, 411 (2009).

[3] A. Brański, Acta Phys. Pol. A 123, 1123 (2013).

[4] E. Żołopa, A. Brański, Acta Phys. Pol. A 125, A155 (2014).

[5] W.P. Rdzanek, K. Szemela, D. Pieczonka, Arch. Acoust. 36, 121 (2011).

[6] W.J. Rdzanek, W.P. Rdzanek, Z. Engel, Acustica 87, 206 (2001).

[7] A. Ozga, Acta Phys. Pol. A 123, 1034 (2013).

[8] M. Jabłoński, A. Ozga, Acta Phys. Pol. A 121, A174 (2012).

[9] J. Wiciak, Arch. Acoust. 32, 265 (2007).

[10] J. Wiciak, Eur. Phys. J. S. T. 154, 229 (2008).

[11] M. Wiciak, R. Trojanowski, Acta Phys. Pol. A 123 , 1048 (2013).

[12] J. Wiciak, R. Trojanowski, Acta Phys. Pol. A $\mathbf{1 2 5}$ A-179 (2014).

[13] M. Wiciak, R. Trojanowski, Acta Phys. Pol. A 125 , A183 (2014).

[14] J. Wiciak, R. Trojanowski, Arch. Acoust. 40, 71 (2015).

[15] W.P. Rdzanek, Wibroakustyka Strukturalna elementów powierzchniowych, Wydawnictwo Uniwersytetu Rzeszowskiego, Rzeszów 2011.

[16] J.W.S. Rayleigh, Theory of Sound, MacMillan, Cambridge 1929 . 